\title{
Espesores críticos de relajación en pozos cuánticos de InGaAs/ GaAs sobre sustratos de GaAs (001) y (111)B
}

\author{
M. GUTIÉRREZ, D. GONZÁLEZ, G. ARAGÓN, J. J. SÁNCHEZ1', I. IZPURA Y R. GARCíA. \\ Dpto. Ciencia de los Materiales e I. M. y Química Inorgánica, Universidad de Cádiz, Apdo. 40, Puerto Real, 11510 Cádiz \\ ${ }^{1}$ Dpto. Ingeniería Electrónica. E. T. S. I. Telecomunicación. Universidad Politécnica de Madrid. Ciudad Universitaria s/n. 28040 Madrid.
}

\begin{abstract}
Se presenta un estudio mediante Microscopía Electrónica de Transmisión (TEM) y Fotoluminiscencia (PL) de espesores críticos de epicapa (CLT) en pozos cuánticos simples de InGaAs/GaAs crecidos sobre substratos de GaAs con orientaciones (001) y (111)B. Los resultados obtenidos demuestran que la disminución de la señal luminiscente del espectro de PL se debe a distintos mecanismos de relajación para cada orientación de sustrato. Así, en sustratos orientados (001), el CLT viene definido por la transición desde un frente de crecimiento plano (2D) a otro ondulado (3D). Sin embargo, la relajación plástica debida a una red de dislocaciones de desajuste (DD) determina el CLT en sustratos orientados (111)B. El análisis por TEM de esta red de dislocaciones muestra la existencia de una nueva configuración de DD distinta a la anteriormente descrita en la bibliografía. La nueva configuración observada permite explicar los resultados experimentales obtenidos para el caso de SQW de InGaAs / GaAs (111)B utilizando los modelos clásicos de CLT de formación de la primera DD.
\end{abstract}

Palabras Clave: espesor crítico, (111)B GaAs, relajación, optoelectrónica

Critical thickness of relaxation in InGaAs/GaAs single quantum well on (001) and (111)B GaAs substrates

A study of critical layer thickness (CLT) in single quantum wells of InGaAs/GaAs on GaAs substrates with (001) and (111)B orientations is presented. The results obtained by Transmission Electronic Microscopy (TEM) and Photoluminescence (PL) show that the decrease of the luminescent signal is due to different relaxation mechanisms for each substrate orientation. Thus, in (001) substrates, the CLT is defined by the transition from a plane growth front (2D) to a rough one (3D). However, the plastic relaxation due to a misfit dislocation array (DD) determines the CLT in (111)B substrates. The analysis by TEM of this dislocation array shows a new DD configuration different to the previously described in the bibliography. The new observed configuration allows us to explain the experimental results obtained for the case of SQW of InGaAs/GaAs (111)B using the classic CLT models of the first DD formation.

Keywords: critical thickness, (111)B GaAs, relaxation, optoelectronics

\section{INTRODUCCIÓN}

La ventana de transmisión óptica situada entre 1-1.3 $\mu \mathrm{m}$ tiene un especial interés en la ingeniería de telecomunicaciones por fibra óptica. Las estructuras de pozos cuánticos (QWs) de capas de InGaAs/GaAs tensadas sobre substratos de GaAs (001) han sido utilizadas por numerosos grupos en la última década. Sin embargo, para altos contenidos de In, la relajación plástica por formación de dislocaciones ha limitado la operatividad de estas estructuras a longitudes de onda de emisión inferiores a $1 \mu \mathrm{m}$. Sin embargo, actualmente el crecimiento sobre sustratos con orientaciones distintas a la (001) ha abierto la posibilidad de obtener estructuras con emisión a mayores longitudes de onda $(\lambda>1 \mu \mathrm{m})$.

Desde hace una década (1), se conoce que los semiconductores III-V poseen un importante campo piezoeléctrico para las orientaciones de sustrato distintas a la orientación habitual (001). De todas las orientaciones, la orientación (111) es la que posee mayor campo piezoeléctrico, pudiendo alcanzar valores del orden de $4 \times 10^{5} \mathrm{~V} / \mathrm{cm}$ (2). La posibilidad de modular externamente este campo piezoeléctrico abre nuevas expectativas para obtener moduladores ópticos y láseres sintonizables. En el caso de heteroestructuras InGaAs/GaAs, el campo piezo- eléctrico produce un desplazamiento de la longitud de onda de emisión hacia el rojo, que puede acercarse a la ventana de transmisión de las fibras ópticas, $1.3 \mu \mathrm{m}$.

Además, hay que sumar la expectativa de obtener mayores espesores críticos de capa para la relajación $\left(\mathrm{CLT}^{\mathrm{R}}\right)$ del desajuste reticular, que permita diseñar nuevas estructuras con mayores contenidos de In (3). Los resultados experimentales para la determinación de $\mathrm{CLT}^{\mathrm{R}}$ realizados con técnicas macroscópicas como Difracción de Electrones de Alta Energía Reflejados (RHEED) (4) o PL (5), muestran esta tendencia. Sin embargo, los modelos teóricos utilizados hasta la fecha para explicar este incremento de CLT $^{\mathrm{R}}$ están basados en el espesor crítico de formación de la primera DD en la intercara $\left(\mathrm{CLT}^{\mathrm{DD}}\right)(4,6)$, por lo que las técnicas macroscópicas no son las más adecuadas para su comprobación.

Hasta la actualidad, existen muy pocos estudios sobre espesor crítico utilizando técnicas experimentales para la determinación directa de las primeras dislocaciones de desajuste( DD). En este sentido, la técnica de Microscopía Electrónica de Transmisión permite esta posibilidad. En este trabajo, se realiza un estudio sistemático para la detección de DD en dos series de estructuras de InGaAs crecidas sobre dos tipos de 
sustratos: 1) orientación (001) y 2) orientación (111)B. Los resultados del análisis servirán para comprobar las predicciones de los modelos teóricos de estimación de espesor crítico en estructuras de InGaAs/GaAs para ambos tipos de sustratos y evaluar las posibilidades de mayores longitudes de emisión en sustratos (111)B.

\section{PROCEDIMIENTO EXPERIMENTAL}

Se ha crecido de forma simultánea sobre sustratos de GaAs (001) y (111)B una serie de estructuras de pozo cuántico simple (SQW) de $\mathrm{In}_{\mathrm{x}} \mathrm{Ga}_{1-\mathrm{x}} \mathrm{As} / \mathrm{GaAs}$ por Epitaxia de Haces Moleculares (MBE) en una máquina RIBER 2300. Los sustratos (111)B presentan una desorientación de $1^{\circ}$ hacia la dirección [211], habitualmente empleada para conseguir epicapas de una buena calidad cristalina. La estructura de las muestras consiste en una capa de $0.3 \mu \mathrm{m}$ de GaAs crecida a una temperatura de $615^{\circ} \mathrm{C}$, seguida de una capa de $\operatorname{In}_{\mathrm{x}} \mathrm{Ga}_{1-\mathrm{x}}$ As de $10 \mathrm{~nm}$ a una temperatura de crecimiento de $500^{\circ} \mathrm{C}$, y finalizando con una capa de GaAs de $0.3 \mu \mathrm{m}$. Las composiciones de la capa de $\mathrm{In}_{\mathrm{x}} \mathrm{Ga}_{1-\mathrm{x}}$ As están en el intervalo de $0.12<x<0.35$.

Las muestras fueron preparadas en visión planar y visión transversal para su estudio por Microscopía Electrónica de Transmisión en un JEOL 1200 EX operando a 120 kV en modo contraste de difracción. Las muestras en visión planar se obtuvieron mediante lijado y posterior ataque químico con peróxido de hidrógeno en medio ácido, mientras que en visión transversal el acabado final se realizó por bombardeo de iones $\mathrm{Ar}^{+}$acelerados a $4.5 \mathrm{kV}$.

Los espectros de Fotoluminiscencia se obtuvieron tanto a temperatura ambiente como a baja temperatura ( $23 \mathrm{~K})$ mediante una fuente láser de He-Ne operando a una longitud de onda de $6328 \AA$ Å. La señal fue detectada usando tanto un fotomultiplicador S1 como un detector de Ge.

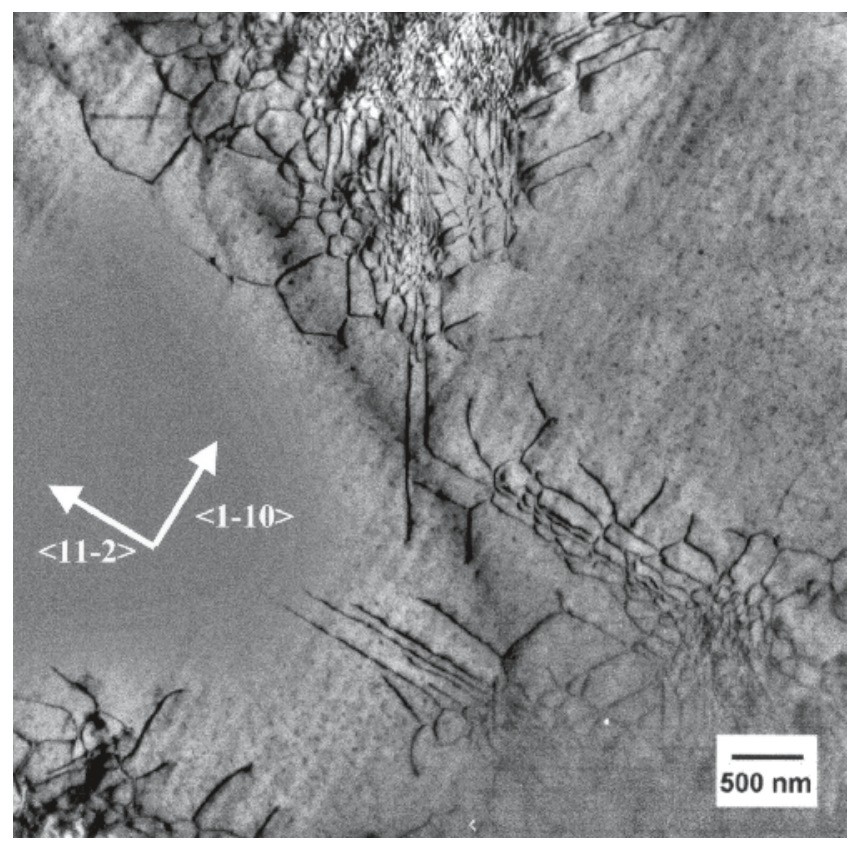

Fig. 1. Morfología de la red DD presente en epicapas InGaAs/GaAs con contenidos de In del 35\% crecidas sobre sustratos GaAs (111)B.

\section{RESULTADOS}

3.1 Estructuras InGaAs/GaAs de pozo cuántico simple crecidas sobre sustratos (111)B

El estudio de este tipo de muestras, realizado mediante Microscopía Electrónica de Transmisión en visión planar (PVTEM), revela que las muestras con un contenido de In inferior al 30\% aparecen libres de defectos reticulares. No obstante, en muestras con contenido de In igual o superior al 31\%, se detecta la presencia de una red de dislocaciones de desajuste de tipo poligonal. La morfología de esta red (Figura 1) es notablemente distinta a la red triangular comúnmente observada para heteroestructuras crecidas en la dirección (111)B para bajo contenido de In $(7,8)$ y que denominaremos dislocaciones tipo I. La red tipo I está constituida por líneas de dislocación que siguen direcciones $\langle 110\rangle$, contenidas en el plano de la intercara, con vectores de Burgers del tipo $1 / 2<110>$ fuera del plano de crecimiento.

Por otro lado, la red poligonal observada es inhomogénea, con áreas donde existe una gran densidad de dislocaciones separadas por otras con muy baja densidad de DD (figura 1). La red de dislocaciones se encuentra formada por dos tipos de líneas siguiendo direcciones $<11 \overline{2}>$ y $<\overline{1} 0>$ contenidas en el plano de la intercara.

El mecanismo de formación de la red poligonal ha sido descrito con anterioridad por los autores (9) para el caso de estructuras InGaAs/GaAs de pozo cuántico múltiple, (MQW). En este tipo de muestras se observó para bajos contenidos de In, la presencia de una configuración de dislocaciones en forma de estrella de tres puntas cuyos brazos forman $120^{\circ}$ entre sí siguiendo direcciones $<11 \overline{2}>$ (Dislocaciones tipo II a). Los vectores de Burgers de estas líneas son del tipo, estando contenidos en el plano de crecimiento a diferencia de las dislocaciones de tipo I. Cuando se incrementa el contenido de In, las estrellas evolucionan formando configuraciones de dislocaciones

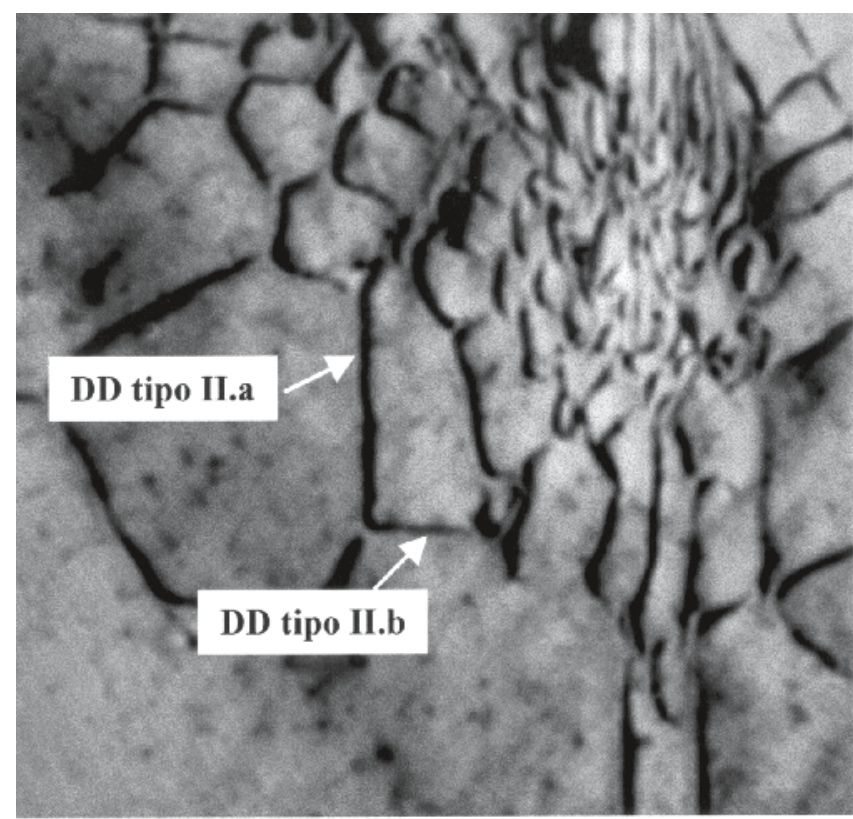

Fig. 2. Red poligonal de dislocaciones de desajuste observada en SQWs de InGaAs/GaAs (111)B donde se distinguen dos tipos de líneas de dislocación: $<110>$ y $<112>$ 
TABLA I. CARACTERÍSTICAS DE LAS DISTINTAS CONFIGURACIONES DE DISLOCACIONES DE DESAJUSTE EN INGAAs/GAAs EN LAS ORIENTACIONES (001) Y (111) B.

\begin{tabular}{|l|c|c|c|c|}
\hline & (001) & (111) TIPO I & (111) TIPO II.A & (111) TIPO II.B \\
\hline Ángulo & $60^{\circ}$ & $60^{\circ}$ & $30^{\circ}$ & $60^{\circ}$ \\
\hline $\mathrm{b}_{\mathrm{r}}$ & 0.50 & 0.30 & 0.50 & 0.87 \\
\hline
\end{tabular}

enmarañadas debido a la interacción de estrellas próximas entre sí. En esta configuración, los brazos de estas estrellas de DD pasaban de direcciones $<1 \overline{1} \overline{2}>$ a direcciones $\langle\overline{1} \overline{1} 0>$, denominando a estas últimas dislocaciones tipo II.b. La semejanza de estos resultados con aquellos encontrados para el caso de MQW (9) con altos contenidos de In, permite determinar la dirección de estas nuevas líneas de dislocación y sus vectores de Burgers en el caso de SQW. Así, la red poligonal está formada por dislocaciones tipo II.a y dislocaciones tipo II.b (figura 2). Ambas líneas poseen vectores de Burgers del tipo $1 / 2$ $<110>$ que se hacen invisibles con las reflexiones 224 . Los vectores de Burgers forman inicialmente $30^{\circ}$ con la línea de dislocación para dislocaciones de tipo II.a, y $60^{\circ}$ en las dislocaciones de tipo II.b.

El cambio del carácter $\alpha$ de las dislocaciones en este tipo de muestras tiene importantes repercusiones en la relajación porque modifica la componente de relajación del desajuste reticular del vector de Burgers, $\boldsymbol{b}_{\boldsymbol{r}}$. En la tabla 1, se relaciona el valor de este parámetro, $\boldsymbol{b}_{r^{\prime}}$ para el caso de dislocaciones perfectas encontradas en el sistema InGaAs/GaAs, en muestras crecidas tanto en la orientación (001) como en la (111). Como se puede observar, la nueva red de dislocaciones tipo II presenta un $\boldsymbol{b}_{r}$ superior a la red de tipo I, llegando incluso a ser superior al valor determinado para las dislocaciones de $60^{\circ}$ presentes cuando la orientación del sustrato es (001). Este resultado, como se verá más adelante, afecta de forma importante a las predicciones de espesor crítico para sustratos (111)B.

Por otro lado, los espectros de PL, obtenidos sobre el mismo grupo de muestras, no muestran cambios significativos en la anchura del pico a media altura de su máximo de emisión (FWHM). Sin embargo, se observa un fuerte descenso en la intensidad del pico (figura 3). Este fenómeno es habitualmente asociado con el inicio de la deformación plástica, consecuencia del descenso de la tensión elástica por la presencia de DD que degradan las propiedades ópticas del material. Los resultados de PL están en buen acuerdo con los de TEM definiendo un $\mathrm{CLT}^{\mathrm{DD}}$ en el intervalo del $24-31 \%$ de In.

\subsection{Estructuras InGaAs/GaAs de pozo cuántico simple creci- das sobre sustratos (001)}

El análisis por PVTEM no detecta la presencia de dislocaciones de desajuste, incluso para contenidos de In superiores al $31 \%$. Sin embargo, los espectros obtenidos mediante PL ponen de manifiesto un cambio brusco del FWHM a partir del $24 \%$ de In, así como en la posición de dicho pico, como se refleja en la figura 4. Existe en este intervalo de composiciones otro fenómeno, distinto a la relajación plástica por formación de DD, que modifica el espectro de emisión de los SQW. En la Figura 5 se muestran micrografías obtenidas por Microscopía Electrónica de Transmisión en sección transversal (CSTEM) de SQW de InGaAs/GaAs con un contenido de In del $31 \%$. Se observa como el SQW no presentan intercaras planas sino un perfil irregular de tipo ondulado. La degradación de la señal luminiscente de las estructuras InGaAs/GaAs crecidas en la orientación (001) se asocia, por tanto, al cambio en la planaridad de las intercaras y no a la presencia de dislocaciones de desajuste, como en el caso de la orientación (111)B.

\section{DISCUSIÓN}

La comparación entre los resultados obtenidos por TEM y PL nos permite constatar que existen diferentes CLTs en la relajación de las epicapas de InGaAs/GaAs. Técnicas macroscópicas como PL permiten determinar el espesor crítico cuando se produce un cambio apreciable en las características de la señal emitida. En el caso de relajación plástica por formación

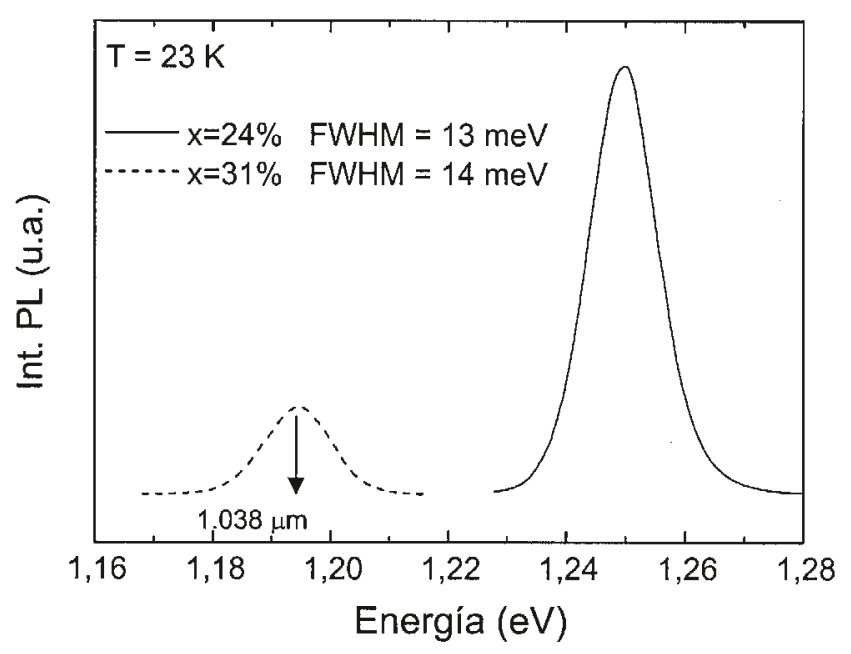

Fig. 3. Espectros de Fotoluminiscencia para SQWs de InGaAs/GaAs con contenidos de In del 24 y $31 \%$, crecidos sobre substratos de GaAs (111)B.

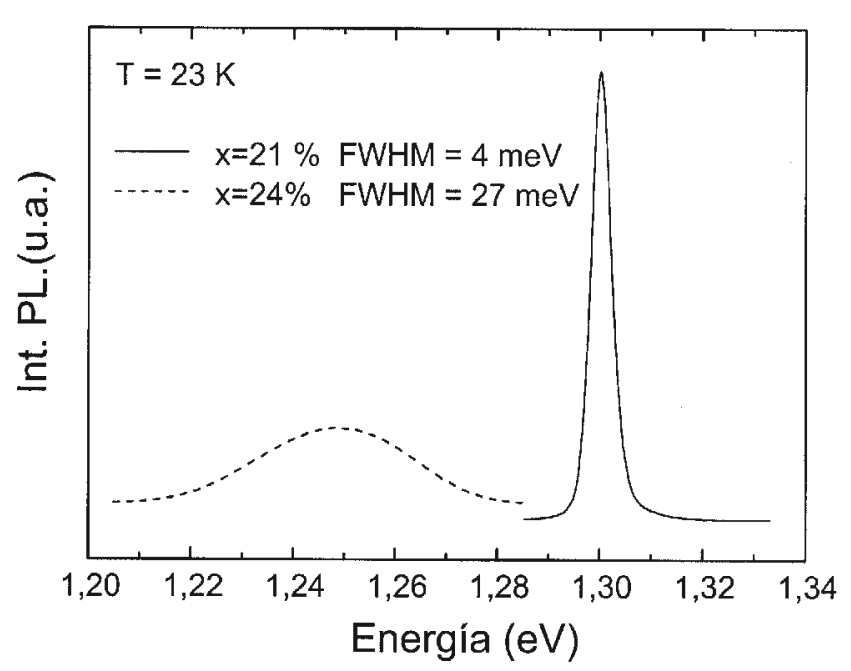

Fig. 4. Espectros de Fotoluminiscencia de InGaAs/GaAs SQWs con contenidos de In del 21 y 24\%, crecidos sobre substratos de GaAs (001). 
de DD, el CLT sólo se detecta con una concentración significativa de DDs que modifiquen de forma macroscópica el estado de deformación del pozo cuántico. No obstante, la formación de una intercara ondulada como consecuencia de un cambio en el modo de crecimiento puede originar también un cambio en la señal de PL, que es sensible a los cambios de espesor de los pozos cuánticos. Este es el caso de los pozos cuánticos de InGaAs crecidos sobre sustratos de GaAs (001) donde para composiciones en torno al $25 \%$ de In, se observa un cambio desde un frente de crecimiento plano (2D) a otro ondulado (3D). Esta rugosidad de la intercara produce un pozo cuántico de espesor variable y, por tanto, un ensanchamiento del pico de emisión así como una disminución de la intensidad del máximo, efectos indeseables para la fabricación de dispositivos. En definitiva, la técnica de PL define un CLT de cambio macroscópico del estado de tensiones del pozo cuántico cuando se modifique la señal luminiscente. Sin embargo, como se ha visto, este cambio no se puede asociar directamente con el inicio de la relajación plástica debida a la formación de DD.

La mayoría de las teorías de espesor crítico existentes en la bibliografía se refieren al cambio en la coherencia de las intercaras como consecuencia de la formación de la primera DD, $\mathrm{CLT}^{\mathrm{DD}}$. Es por ello que únicamente técnicas microscópicas, como TEM, son las adecuadas para verificar las predicciones de los modelos teóricos de espesor crítico. En este sentido, el modelo de Matthews y Blakeslee (10) ha sido utilizado con éxito para predecir el CLT ${ }^{\mathrm{DD}}$ en pozos cuánticos simples en la orientación (001) (11),(12). Para el caso de un crecimiento en un plano $(h, k, l)$, el CLT ${ }^{\mathrm{DD}}$ para un SQW viene dado por la expresión (6):

$$
h_{c}=\frac{\mu_{h k l} b^{2}\left(1-v_{h k l} \cos ^{2} \alpha\right)}{2 M_{h k l} b_{r}} \ln \left(\frac{\gamma h_{c}}{b}\right)
$$

donde $\mu, v$, y $M$ son el módulo de cizalla, de Poisson y biaxial de Young, respectivamente, $\alpha$ el ángulo entre la línea de la dislocación y el vector de Burgers y $\gamma$ es un factor relacionado con el radio del núcleo de la dislocación, para el que tomaremos el valor usual de 4 (13).

En la figura 6, se representa el CLT ${ }^{\mathrm{DD}}$ en función del contenido de In para SQW de InGaAs / GaAs con las distintas confi-

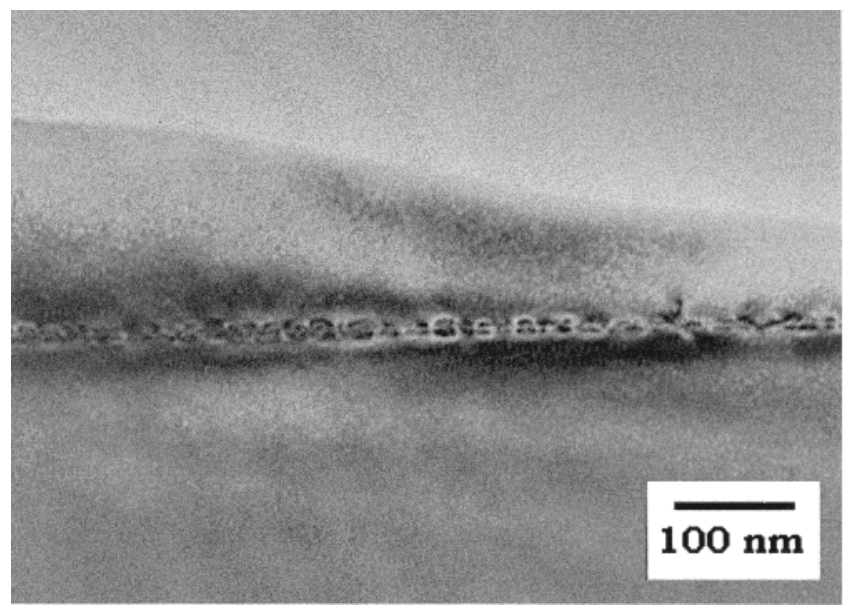

Fig. 5. Micrografía de CSTEM de $\operatorname{In}_{\mathrm{x}} \mathrm{Ga}_{1-\mathrm{x}} \mathrm{As} / \mathrm{GaAs}(001) \mathrm{SQW}(\mathrm{x}=0,31)$ con la reflexión 220 donde se observa la presencia de una intercara ondulada. guraciones de DD observadas en ambas orientaciones de sustratos. Se puede apreciar que la nueva configuración de dislocaciones de tipo II, observada para contenidos de In superiores al $25 \%$, rebaja de forma considerable las expectativas de CLT ${ }^{D D}$ sugeridas por Anan (4) que utilizaba la configuración de dislocaciones tipo I. Además, las dislocaciones tipo II.b presentan un CLT $^{\mathrm{DD}}$ menor incluso que las dislocaciones observadas en sustratos (001). No obstante, estudios realizados en MQW (9) de composiciones análogas, nos ha permitido conocer que la nueva red se origina a partir de una configuración en estrella de dislocaciones de tipo II.a y, por tanto, define el CLT ${ }^{\mathrm{DD}}$ para epicapas de InGaAs/GaAs (111)B de alto contenido de In. Como se observa en la Figura 6, los resultados experimentales obtenidos por TEM se ajustan muy bien a las predicciones teóricas para el caso de dislocaciones tipo II.a.

Según estos resultados, los CLT DD para epicapas de alto contenido de In en sustratos (001) y (111)B son comparables $\mathrm{y}$, en consecuencia, no se cumplirían las expectativas de mayores CLT $^{\mathrm{R}}$ para sustratos (111)B. Sin embargo, como se ha comentado, el $\mathrm{CLT}^{\mathrm{R}}$ no está definido de forma exclusiva por el inicio de la relajación plástica mediante DD. En sustratos (001), el CLT ${ }^{\mathrm{R}}$ se produce por un cambio en el modo de crecimiento que atrasa el CLT ${ }^{\mathrm{DD}}$, no observándose DD en muestras con un contenido de In del $31 \%$. Por tanto, en la dirección (001), para alto contenido de In existen dos tipos de CLTs: el primero, menor, relacionado con el cambio a un modo crecimiento tridimensional y el segundo, mayor, relacionado con la formación de las primeras DD. En cambio, en sustratos (111)B, el CLT ${ }^{\mathrm{R}}$ se corresponde con el CLT ${ }^{\mathrm{DD}}$ al no observarse cambios en el modo de crecimiento. Así, el retraso en el cambio del modo de crecimiento de las muestras crecidas sobre sustratos (111)B respecto a las crecidas sobre sustratos (001) supone una ventaja no esperada que mantiene las expectativas de mayores longitudes de emisión en este tipo de estructuras.

Se ha observado que los sustratos (111)B presentan un CLT $^{\mathrm{R}}$ mayor que los sustratos (001). Sin embargo, las causas son diferentes a las predichas en la bibliografía donde únicamente se utilizaban modelos de formación de DD. El conocimiento de los mecanismos de relajación abre puertas para conseguir dispositivos de InGaAs/GaAs (111)B que alcancen longitudes de emisión de $1.1 \mu \mathrm{m}$.

Fig. 6. Representación del CLT ${ }^{\mathrm{DD}}$ de las distintas configuraciones de DD descritas en la Tabla I en función del contenido de In de SQW de InGaAs/GaAs.

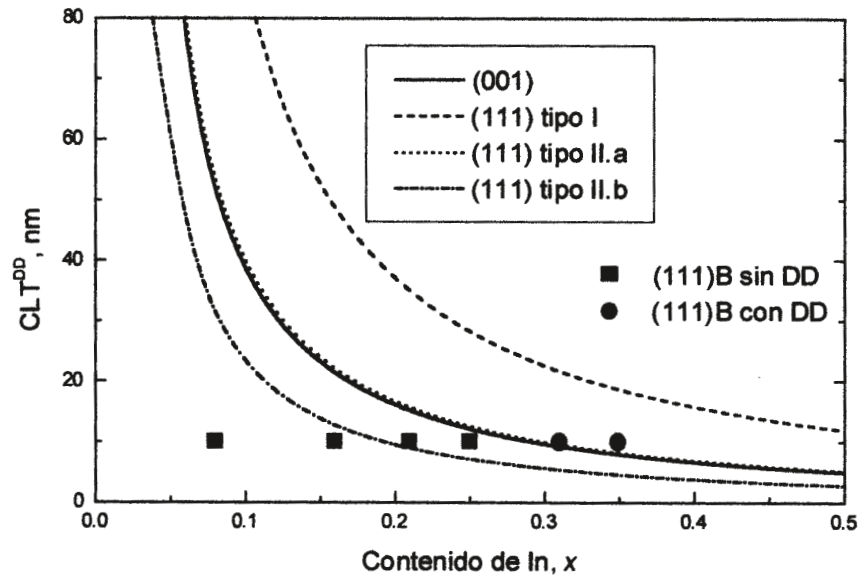




\section{CONCLUSIONES}

La relajación del desajuste reticular en $\mathrm{SQW}$ de $\operatorname{In}_{\mathrm{x}} \mathrm{Ga}_{1-\mathrm{x}} \mathrm{As} /$ GaAs con alto contenido de In ha sido estudiada para dos orientaciones de sustrato: (001) y (111)B. Se ha observado que el espesor crítico de relajación está gobernado por distintos fenómenos dependiendo de la orientación del sustrato. En la orientación (111)B, el CLT viene definido por la formación de una nueva red de DD. Esta nueva red, diferente a la observada para bajo contenido de In, presenta un $\mathrm{CLT}^{\mathrm{DD}}$ comparable al predicho para la orientación (001). Sin embargo, en la dirección (001) se determina un CLT relacionado con el cambio a un modo de crecimiento tridimensional, que es menor que el relacionado con la formación de las primeras DD. Por tanto, aunque con un CLT inferior al predicho en modelos previos, el crecimiento en la dirección (111)B permite la incorporación de un mayor contenido de In en heteroestructuras InGaAs/GaAs respecto a la orientación (001) y presenta una mayor potencialidad para la fabricación de dispositivos que operen a una longitud de onda comprendida entre 1.0 y $1.3 \mu \mathrm{m}$.

\section{AGRADECIMIENTOS}

El presente trabajo está subvencionado por el proyecto de la CICYT TIC98-0826 y por la Junta de Andalucía (PAI TEP0120). El estudio de Microscopía Electrónica de Transmisión se realizó en los servicios Centrales de Ciencia y Tecnología de la Universidad de Cádiz.

\section{BIBLIOGRAFÍA}

1. D. L. Smith. "Strain generated electric fields in (111) growth axis strained layer superlattices". Sol. State Commun., 57, 919-921 (1986).

2. E. A. Caridi, T . Y. Chang, K. W. Goosen y L. F. Eastman. “Direct demonstration of a misfit strain generated electric field in a [111] growth axis zinc-blende heterostructure". Appl. Phys. Lett., 56, 659-661 (1990).

3. F. Calle, A. L. Alvárez, A. Sacedón y E. Calleja. "Strain diagnosis of (001) and (111) InGaAs layers by optical techniques". Phys. Stat. Solid (a), 152, 201-209 (1995).

4. T. Anan, K. Nishi y S. Sugou. "Critical layer thickness on (111)B oriented InGaAs/GaAs heteroepitaxy", Appl. Phys. Lett., 60, 3159-3161, (1992).

5. A. Sacedón, F. Calle, A. L. Alvarez, E. Calleja, E. Muñoz, R. Beanland y P. Goodhew. "Relaxation of InGaAs layers grown on (111)B GaAs". Appl. Phys. Lett., 65, 3212-3214 (1994).

6. F. Colson y D. Dunstan. "Equilibrium critical thickness of epitaxial strained layers in the $\{111\}$ orientations". J. Appl. Phys., 81, 2898-2900 (1997).

7. T. E. Mitchell y O. Unal. "Dislocation Arrangements in GaAs/GaInAs multilayers grown on (001), (111) and (112) substrates". J. Electron. Mater., 20, 723734 (1991).

8. S. P. Edirisingehe, A. E. Staton-Bevan y R. Grey. "Relaxation mechanisms in single InGaAs epilayers grown on misoriented GaAs (111)B substrates". J. Appl. Phys., 82, 4870-4876 (1997).

9. M. Gutiérrez, D. González, G. Aragón, J. J. Sánchez, I. Izpura y R. García "New relaxation mechanisms in InGaAs/GaAs (111) multiple quantum well". Microelectron. J., 30, 467-470 (1999).

10. J. M. Matthews y A. E. Blakeslee. "Defects in epitaxial multilayers I. Misfit dislocations". J. Crystal Growth, 27, 118-123 (1974).

11. Y. C. Chen y P. K. Bhattacharya. "Determination of critical layer thickness and strain tensor in InGaAs/GaAs quantum well structures by x-ray-difraction", J. Appl. Phys., 73, 7389-7394 (1993).

12. T. G. Anderson, Z. G. Chen, V. D. Kulakovskii, A. Uddin y J. T. Vallin. "Variation of the critical layer thickness with In content in strained InGaAs / GaAs quantum wells grown by molecular Beam epitaxy", Appl. Phys. Lett., 51, 752-754 (1987). 